\title{
ON THE DOUBLE SUSPENSION HOMOMORPHISM AT ODD PRIMES
}

\author{
BY
}

\author{
J. R. HARPER AND H. R. MILLER
}

\begin{abstract}
We work with the $E_{1}$-term for spheres and the stable Moore space, given by the $\Lambda$-algebra at odd primes. Writing $W(n)=\Lambda(2 n+1) / \Lambda(2 n-1)$ and $M(0)$ $=H_{*}\left(S^{0} \cup_{p} e^{1}\right)$, we construct compatible maps $f_{n} \cdot W(n) \rightarrow M(0) \tilde{\otimes} \Lambda$ and prove the Metastability Theorem: in homology $f_{n}$ induces an isomorphism for $\sigma<$ $2\left(p^{2}-1\right)(s-2)+p q n-2 p-2$ where $\sigma=$ stem degree, $s=$ homological degree resulting from the bigrading of $\Lambda$ and $q=2 p-2$. There is an operator $v_{1}$ corresponding to the Adams stable self-map of the Moore space and $v_{1}$ extends to $W(n)$. A corollary of the Metastability Theorem and the Localization Theorem of the second author is that the map $f_{n}$ induces an isomorphism on homology after inverting $v_{1}$.
\end{abstract}

In this paper we carry over to odd primes $p$ certain ideas of Mark Mahowald [7]. Our goal is to establish a metastable range for the $\bmod p$ unstable Adams $E_{2}$-term for an odd-dimensional sphere. To be more specific, visualize the $E_{2}$-term displayed in the $(\sigma, s)$-plane, where $\sigma=$ stem degree and $s=$ homological degree. Then we show that above a line of slope $1 / 2\left(p^{2}-1\right)$ and $\sigma$-intercept $2 p(p-1) n$ minus a constant independent of $n$, the obstructions to double suspension being an isomorphism lie in the stable $E_{2}$-term for the mod $p$ Moore space $S^{0} \cup_{p} e^{1}$.

As in [7], we work with the $E_{1}$-term for spheres and for the stable Moore space given by the "lambda algebra" ([2], as corrected in [3]). This material is reviewed in $\S 1$. Thus, we have complexes $\Lambda(2 n+1)$ and inclusions $\Lambda(2 n-1) \subset \Lambda(2 n+1)$, such that $H(\Lambda(2 n+1))$ is the $E_{2}$-term for $S^{2 n+1}$ and the inclusion induces the $E_{2}$-level of a map of spectral sequences compatible at $E_{\infty}$ with the double suspension homomorphism. Also, if $H_{*}\left(S^{0} \cup_{p} e^{1}\right)=M(0)$, there is a differential bigraded algebra $M(0) \tilde{\otimes} \Lambda$ serving as a stable Adams $E_{1}$-term for the Moore space. We shall always index these complexes by homological degree $s$, written above, and stem degree $\sigma$, written below. Let $W(n)=\Lambda(2 n+1) / \Lambda(2 n-1)$, indexed so that the "lower left-hand corner" occurs at $(s, \sigma)=(0,0)$. Thus, there is a long exact sequence (in which $q=2(p-1)$ )

$$
\cdots \rightarrow H_{\sigma-q n+2}^{s-2} W(n) \rightarrow H_{\sigma}^{s} \Lambda(2 n-1) \rightarrow H_{\sigma}^{s} \Lambda(2 n+1) \rightarrow H_{\sigma-q n+1}^{s-1} W(n) \rightarrow \cdots .
$$

Our initial technical result (in \$2) is an explicit and convenient form of the differential in $W(n)$. This shows immediately that $W(n)$ includes into $W(n+1)$ and

Received by the editors December 15, 1980 and, in revised form, August 17, 1981. 1980 Mathematics Subject Classification. Primary 55P40, 55T15; Secondary 55Q25, 55U99.

${ }^{1}$ The authors were supported in part by the NSF. 
that they all map compatibly to $M(0) \tilde{\otimes} \Lambda$. Our main result is then the

METASTABILITY THEOREM. The map in homology induced by $W(n) \rightarrow M(0) \tilde{\otimes} \Lambda$ is an isomorphism for

$$
\sigma<2\left(p^{2}-1\right)(s-2)+p q n-2 p-2
$$

and an epimorphism for

$$
\sigma \leqslant 2\left(p^{2}-1\right)(s-1)+p q n-2 p-2 .
$$

This result should be seen in the light of the

LOCALIZATION THEOREM [8]. There is an algebra map

$$
l: H(M(0) \tilde{\otimes} \Lambda) \rightarrow P\left[v_{1}\right] \otimes E\left[h_{i, 0}: i \geqslant 1\right] \otimes P\left[b_{i, 0}: i \geqslant 1\right]
$$

where $\left|v_{1}\right|=(1, q),\left|h_{i, 0}\right|=\left(1,2\left(p^{i}-1\right)-1\right)$, and $\left|b_{i, 0}\right|=\left(2,2 p\left(p^{i}-1\right)-2\right)$; and $l$ is an isomorphism in bidegrees $(s, \sigma)$ for which $\sigma \leqslant\left(p^{2}-p-1\right)(s+1)$.

Thus one may say that at the Adams $E_{2}$-level, the obstructions to double suspension being an isomorphism are themselves stable invariants, for filtration sufficiently high relative to stem degree; and that the groups in which these obstructions occur have been computed in a somewhat smaller range.

The operator $v_{1}$ occurring in the Localization Theorem lifts to a self-map of $W(n)$; indeed, for $n>1, v_{1}$ carries $W(n)$ into $W(n-1)$. The Metastability Theorem then implies that, for all $n \geqslant 1, H(W(n)) \rightarrow H(M(0) \tilde{\otimes} \Lambda)$ is an isomorphism after inverting $v_{1}$; and the Localization Theorem computes the resulting groups.

The proof of the Metastability Theorem is carried out in $\$ \S 3$ and 4. It follows Mahowald's strategy, relying however on the mod $p$ vanishing line of [9].

While no stem by stem calculations are performed here, nevertheless the differential structure of $W(n)$ (stated as Proposition 2.10) is the basis for a convenient and systematic inductive calculation of the unstable Adams $E_{2}$-terms for spheres. These calculations find an application in [4], where they are used to evaluate obstructions to $H$-structures on sphere bundles over spheres.

The authors are grateful to Mahowald for explaining his ideas to them. Their hope is that this exposition of the simpler odd-primary case will make his work accessible to a wider audience.

Finally, we note that F. R. Cohen [11] has constructed geometrical analogues of certain parts of this work.

1. The lambda algebra for odd primes. In this section, we shall record certain properties of the lambda algebra at an odd prime $p$. See [3 and 10] for further description and techniques of calculation. The only new result in this section is Proposition 1.18.

Here and throughout the paper, we index bigraded objects by (filtration or homological degree, stem degree $)=(s, \sigma)$, and write $X_{\sigma}^{s}$ for a given bidegree. The "complementary degree" $t$ satisfies $\sigma=t-s$. Signs are invariably controlled by stem degree $\sigma$. 
Fix an odd prime $p$, and let $q=2(p-1)$. The lambda algebra $\Lambda$ is the associative bigraded $\mathbf{F}_{p}$-algebra generated by

$$
\begin{array}{ll}
\lambda_{i} \text { of bidegree }(1, q i-1), & i \geqslant 1, \\
\mu_{i} \text { of bidegree }(1, q i), & i \geqslant 0,
\end{array}
$$

subject to the following "Adem" relations. Define elements

$$
\begin{gathered}
c_{k j}=(-1)^{j+1}\left(\begin{array}{c}
(p-1)(k-j)-1 \\
j
\end{array}\right), \\
d_{k j}=(-1)^{j}\left(\begin{array}{c}
(p-1)(k-j) \\
j
\end{array}\right)
\end{gathered}
$$

of $\mathbf{F}_{p}$, where the binomial coefficients are subject to the usual conventions. In particular,

$$
\begin{array}{ll}
c_{k j}=0 & \text { for } p j \geqslant(p-1) k, \\
d_{k j}=0 & \text { for } p j>(p-1) k .
\end{array}
$$

Then, for $i>0, k \geqslant 0$ in (1.7) and (1.8), and $i \geqslant 0, k \geqslant 0$ in (1.9) and (1.10):

$$
\begin{gathered}
\lambda_{i} \lambda_{p i+k}=\sum_{j} c_{k j} \lambda_{i+k-j} \lambda_{p i+j}, \\
\lambda_{i} \mu_{p i+k}=\sum_{j}\left(c_{k j} \lambda_{i+k-j} \mu_{p i+j}+d_{k j} \mu_{i+k-j} \lambda_{p i+j}\right), \\
\mu_{i} \lambda_{p i+k+1}=\sum_{j} c_{k j} \mu_{i+k-j} \lambda_{p i+j+1}, \\
\mu_{i} \mu_{p i+k+1}=\sum_{j} c_{k j} \mu_{i+k-j} \mu_{p i+j+1} .
\end{gathered}
$$

A monomial in the $\lambda$ 's and $\mu$ 's is called admissible if

(1.11) whenever $\lambda_{i} \lambda_{j}$ or $\lambda_{i} \mu_{j}$ occurs, we have $j<p i$, and

(1.12) whenever $\mu_{i} \lambda_{j}$ or $\mu_{i} \mu_{j}$ occurs, we have $j \leqslant p i$.

The relations (1.7)-(1.10) show that the admissible monomials form a basis for $\Lambda$.

The algebra $\Lambda$ has a differential $d$, defined by

$$
\begin{gathered}
d \lambda_{k}=\sum_{j} c_{k j} \lambda_{k-j} \lambda_{j}, \\
d \mu_{k}=\sum_{j}\left(c_{k j} \lambda_{k-j} \mu_{j}+d_{k j} \mu_{k-j} \lambda_{j}\right) .
\end{gathered}
$$

As a differential algebra, $\Lambda$ respects a third grading, the Cartan degree, given by the number of $\mu$ 's present.

The complex $\Lambda$ is filtered by subcomplexes $\Lambda(n), n \geqslant 0$, where $\Lambda(n)$ is the submodule with basis given by the admissible monomials beginning with

(1.15) $\lambda_{i}$ with $i \leqslant m$ or $\mu_{i}$ with $i<m$ if $n=2 m$,

(1.16) $\lambda_{i}$ with $i \leqslant m$ or $\mu_{i}$ with $i \leqslant m$ if $n=2 m+1$.

These submodules have the important property that

$$
\Lambda(n)_{\sigma} \Lambda(n+\sigma) \subseteq \Lambda(n) .
$$

In particular, $\Lambda(n)$ is a subalgebra. 
As a first step in an analysis of the product in $\Lambda$, as it relates to these subcomplexes, we have the following result. Recall that $q=2(p-1)$.

Proposition 1.18. The subcomplexes $\Lambda(n)$ satisfy

$$
\begin{gathered}
\lambda_{i} \Lambda(m) \subseteq \Lambda(m-q i) \text { for } m \geqslant 2 p i, i>0, \\
\mu_{i} \Lambda(2 m+1) \subseteq \Lambda(2 m-q i-1) \text { for } m>p i, i \geqslant 0 .
\end{gathered}
$$

Proof. Define the moment of the monomial $\nu_{i_{1}} \cdots \nu_{i_{s}}$ (where $\nu$ denotes either $\lambda$ or $\mu)$ to be the integer $i_{1}+2 i_{2}+\cdots+s i_{s}$. Then we observe

(1.19) the relations (1.7)-(1.10) express an inadmissible monomial as a sum of admissible monomials of strictly smaller moment; and

(1.20) monomials of a fixed length are admissible for sufficiently small moment.

The proof is now by a double induction on the length and moment of the product. The induction starts since the result is true for the length 1 products $\lambda_{i} \cdot 1, \mu_{i} \cdot 1$, and is true for any sum of monomials $\nu$ for which $\lambda_{i} \nu$ or $\mu_{i} \nu$ is admissible.

Take, for example, a product $\lambda_{i} \nu$ with $\nu$ an admissible monomial in $\Lambda(m)$. We must consider two cases, depending on the leading letter in $\nu$. Suppose, for example, that $\nu=\mu_{p i+k} \alpha$, with $\alpha \in \Lambda(2 p(p i+k)+1)$. We may as well suppose $m=$ $2(p i+k)+1$. If $k=0$, the statement holds, since $\lambda_{i} \mu_{p i}=0$; so suppose $k>0$. Then we claim that

$$
\lambda_{i} \nu \in \Lambda(2(i+k)+1)
$$

Now

$$
\lambda_{i} \mu_{p i+k} \alpha=\sum_{j}\left(c_{k j} \lambda_{i+k-j} \mu_{p i+j} \alpha+d_{k j} \mu_{i+k-j} \lambda_{p i+j} \alpha\right) .
$$

If each monomial on the right is admissible, the proof in this case is complete (and we see from this that the inclusion relations cannot be improved). If not, take an inadmissible term, say $\mu_{p i+j} \alpha$. Since (1.5) shows that $j<k$, our inductive hypothesis on shorter products implies that

$$
\mu_{p i+j} \alpha \in \Lambda(2 p(p i+k)-q(p i+j)-1) \subseteq \Lambda(2 p(i+k-j)+2 j+1) .
$$

Next, expanding $\mu_{p i+j} \alpha$ into a sum $\Sigma \nu_{l}$ of admissible monomials results in terms of smaller moment, so the moment of each term $\lambda_{i+k-j} \nu_{l}$ is less than that of $\lambda_{i} \nu$. Since $2 p(i+k-j)+2 i+1>2 p(i+k-j)$, our inductive hypothesis on smaller moment implies that

$$
\begin{aligned}
\lambda_{i+k-j} \mu_{p i+j} \alpha & \in \Lambda(2 p(i+k-j)+2 j-q(i+k-j)+1) \\
& =\Lambda(2(i+k)+1)
\end{aligned}
$$

as desired.

The other terms in (1.22), and the other cases of the Adem relations, are handled by four other similar computations.

REMARK 1.23. The relations (1.17) can be proved by inductions on length in $\Lambda(n)_{\sigma}$ using Proposition 1.18.

REMARK 1.24. The case $i=0$ of the inclusion relations asserts that, for $n \geqslant 1$, $\mu_{0} \Lambda(2 n+1) \subseteq \Lambda(2 n-1)$. This is an $E_{1}$-version of the Cohen-Moore-Neisendorfer theorem asserting that $p \pi_{*}\left(S_{(p)}^{2 n+1}\right)$ is contained in the image of the double suspension homomorphism. It is tempting to try to find geometric analogues of the other 
relations. For example, if $m \geqslant p$ and $\gamma \in \pi_{k} S^{2 m+1}$, is $\gamma \circ \alpha_{1} \in \pi_{k} S^{2 m-q+2}$ a suspension? (We thank the referee for pointing out the absurdity of an earlier geometric formulation, and for leading us to the following interesting case of this question. Take $p=3$ and $\gamma=\beta_{2} \in \pi_{35}\left(S^{9}\right)$. Then $\beta_{2} \circ \alpha_{1} \in \pi_{35}\left(S^{6}\right)$ is indeed a suspension.)

2. The "fiber of double suspension". In this section, we study the complexes $W(n)=\Lambda(2 n+1) / \Lambda(2 n-1)$ representing the fibers of the double suspension maps $S^{2 n-1} \rightarrow \Omega^{2} S^{2 n+1}$. We analyze their differential and show that they filter the stable lambda $E_{1}$-term for a mod $p$ Moore space. Finally, we indicate how the Adams self-map of the Moore space appears in this context.

We will often use the notation $a X$ to denote the tensor product of the one-dimensional $\mathbf{F}_{p}$-module $\langle a\rangle$ generated by $a$ (in a given bidegree) with the bigraded complex $X$. This has the usual sign-changing effect on the differential in $X$.

Note that

$$
\begin{aligned}
& \Lambda(2 n)=\Lambda(2 n-1) \oplus \lambda_{n} \Lambda(2 p n-1), \\
& \Lambda(2 n+1)=\Lambda(2 n) \oplus \mu_{n} \Lambda(2 p n+1) .
\end{aligned}
$$

Together with the observation that $d \lambda_{n} \in \Lambda(2 n-1)$ and $d \mu_{n} \in \Lambda(2 n)$, this implies that we have short exact sequences of complexes

$$
\begin{aligned}
& 0 \rightarrow \Lambda(2 n-1) \rightarrow \Lambda(2 n) \stackrel{h}{\rightarrow} \bar{\lambda}_{n} \Lambda(2 p n-1) \rightarrow 0, \\
& 0 \rightarrow \Lambda(2 n) \rightarrow \Lambda(2 n+1) \stackrel{\vec{h}}{\rightarrow} \bar{\mu}_{n} \Lambda(2 p n+1) \rightarrow 0 .
\end{aligned}
$$

Here $\left|\bar{\lambda}_{n}\right|=(0,0),\left|\bar{\mu}_{n}\right|=(0,1)$, and the "Hopf invariants" $h, \bar{h}$ have bidegree $(-1,-q n+1)$ and are defined on admissible monomials by

$$
\begin{array}{ll}
h(\nu)=0 & \text { unless } \nu=\lambda_{n} \alpha, \text { when } h(\nu)=\bar{\lambda}_{n} \alpha, \\
\bar{h}(\nu)=0 & \text { unless } \nu=\mu_{n} \alpha, \text { when } \bar{h}(\nu)=\bar{\mu}_{n} \alpha .
\end{array}
$$

RemarK 2.7. These exact sequences, together with the "initial conditions"

$$
\Lambda(0)=\langle 1\rangle, \quad \Lambda(n)^{s}=0 \quad \text { for } s<0
$$

determine the vector space $\Lambda(n)$ for all $n \geqslant 0$ by induction on $n$ and $s$.

These sequences fit into a diagram

$$
\begin{aligned}
& \begin{array}{lll}
0 & 0 & 0 \\
\downarrow & \downarrow & \downarrow
\end{array} \\
& 0 \rightarrow \Lambda(2 n-1) \rightarrow \Lambda(2 n) \quad \stackrel{h}{\rightarrow} \bar{\lambda}_{n} \Lambda(2 p n-1) \rightarrow 0 \\
& \downarrow \quad \downarrow \quad \downarrow \\
& \begin{array}{ccccccc}
0 \rightarrow \Lambda(2 n-1) & \rightarrow & \Lambda(2 n+1) & \stackrel{\hat{h}_{1}}{\rightarrow} & W(n) & \rightarrow & 0 \\
\downarrow & & \downarrow \bar{h} & & \downarrow & & \\
0 & \rightarrow & \bar{\mu}_{n} \Lambda(2 p n+1) & \rightarrow & \bar{\mu}_{n} \Lambda(2 p n+1) & \rightarrow & 0 \\
& & \downarrow & & \downarrow & & \\
& & 0 & & 0 & &
\end{array}
\end{aligned}
$$


in which $W(n)$ is defined by the middle row, and $\left|\hat{h}_{1}\right|=(-1,-q n+1)$. We have adjusted bidegrees so that $W(n)$ begins in $(0,0)$ with $\bar{\lambda}_{n}$; the second index might be called the metastable stem. We have a natural additive splitting

$$
W(n) \cong \bar{\lambda}_{n} \Lambda(2 p n-1) \oplus \bar{\mu}_{n} \Lambda(2 p n+1)
$$

and our next result determines the differential in these terms.

Proposition 2.10. Let $n>0, \alpha \in \Lambda(2 p n-1)$, and $\nu \in \Lambda(2 p n+1)$. Then in $W(n)$,

$$
d\left(\bar{\lambda}_{n} \alpha+\bar{\mu}_{n} \nu\right)=\bar{\lambda}_{n}\left(d \alpha+\mu_{0} \nu\right)-\bar{\mu}_{n} d \nu
$$

Proof. For this we must show that

$$
d\left(\lambda_{n} \alpha+\mu_{n} \nu\right)=-\lambda_{n}\left(d \alpha+\mu_{0} \nu\right)+\mu_{n} d \nu \bmod \Lambda(2 n-1) .
$$

The sign reversal occurs because $\Lambda(2 n+1) \rightarrow W(n)$ has odd degree. From (1.14) we have, for example

$$
d\left(\mu_{n} \nu\right)=-\lambda_{n} \mu_{0} \nu+\sum_{j>0}\left(c_{n j} \lambda_{n-j} \mu_{j} \nu+d_{n j} \mu_{n-j} \lambda_{j} \nu\right)+\mu_{n} d \nu
$$

Since $p n>p j$, Proposition 1.18 implies that $\mu_{j} \nu \in \Lambda(2 p n-1-q j)$ and since $2 p n-1-q j=2 p(n-j)+2 j-1>2 p(n-j)$ for $j>0$, Proposition 1.18 gives

$$
\lambda_{n-j} \mu_{j} \nu \in \Lambda(2 p(n-j)+2 j-1-q(n-j))=\Lambda(2 n-1) .
$$

Similarly, we find that the terms $\mu_{n-j} \lambda_{j} \nu$ in (2.11) lie in $\Lambda(2 n-1)$; and analogous calculations for $d\left(\lambda_{n} \alpha\right)$ give the result.

COROllary 2.12. The map $W(n) \rightarrow W(n+1)$ given by

$$
\bar{\lambda}_{n} \alpha+\bar{\mu}_{n} \nu \rightarrow \bar{\lambda}_{n+1} \alpha+\bar{\mu}_{n+1} \nu
$$

of bidegree $(0,0)$ respects differentials and is an isomorphism for metastable stem degree $\sigma<p q n-1$.

Let $A^{*}$ denote the $\bmod p$ Steenrod algebra, graded nonpositively. For any spectrum $X, H_{*}(X)$ is naturally a right $A^{*}$-module with the property that any element lies in a finite submodule. For such an $A^{*}$-module $N$, Bousfield and $\mathrm{Kan}$ [3] define a twisted differential on $N \otimes \Lambda$ by

$$
d(x \otimes \nu)=(-1)^{|x|} \sum_{i>0} x P^{i} \otimes \lambda_{i} \nu+\sum_{i \geqslant 0} x \beta P^{i} \otimes \mu_{i} \nu+(-1)^{|x|} x \otimes d \nu
$$

Write $N \tilde{\otimes} \Lambda$ for the resulting complex. Then, for a spectrum $X, H_{*}(X) \tilde{\otimes} \Lambda$ is an $E_{1}$-term for the Adams spectral sequence abutting to $\pi_{*}(X)$.

Let $M(0)=\langle x, y\rangle$ denote the homology of the mod $p$ Moore spectrum $V(0)=S^{0}$ $\cup_{p} e^{1}$, with $y \beta=x$. Then (omitting “ $\otimes$ ") in $M(0) \tilde{\otimes} \Lambda$,

$$
d(x \alpha+y \nu)=x\left(d \alpha+\mu_{0} \nu\right)-y d \nu .
$$

Comparing with Proposition 2.10, we see that the inclusions $W(n) \rightarrow M(0) \tilde{\otimes} \Lambda$ by

$$
\bar{\lambda}_{n} \alpha+\bar{\mu}_{n} \nu \mapsto x \alpha+y \nu
$$

provide us with a differential filtration of $M(0) \tilde{\otimes} \Lambda$. The map (2.15) is clearly an isomorphism for $\sigma<p q n-1$; so $M(0) \tilde{\otimes} \Lambda=\cup_{n \geqslant 1} W(n)$. 
An important feature of the mod $p$ Moore space is the presence of a self-map $\phi$ : $\Sigma^{q} V(0) \rightarrow V(0)$ inducing an isomorphism in $K$-theory. This map was studied by Adams [1] and is intimately connected with the image of the $J$-homomorphism. It is characterized by the homology of its mapping cone $V(1)$ as an $A^{*}$-module. Writing $\bar{x}$ and $\bar{y}$ for the pullback of $\Sigma^{q+1} x$ and $\Sigma^{q+1} y$, we have $\bar{y} \beta=-\bar{x}, \bar{x} P^{1}=y$, and $y \beta=x$. By the Geometric Boundary Lemma [5], $\phi$ is represented by the algebraic boundary homomorphism associated to the short exact sequence

$$
0 \rightarrow H_{*} V(0) \rightarrow H_{*} V(1) \rightarrow H_{*} \Sigma^{q+1} V(0) \rightarrow 0 .
$$

Using the relations $\mu_{0} \lambda_{1}=0, \mu_{0} \mu_{1}=0, d \lambda_{1}=0, d \mu_{1}=-\lambda_{1} \mu_{0}$ we find that this boundary homomorphism is represented by the degree $q+1$ chain map $v_{1}: M(0)$ $\tilde{\otimes} \Lambda \rightarrow M(0) \tilde{\otimes} \Lambda$ given by $v_{1}: x \alpha+y \nu \mapsto-y\left(\lambda_{1} \alpha+\mu_{1} \nu\right)$.

The algebraic content of these remarks forms the first part of the next proposition, in which we write $\bar{\mu}_{0}: W(n+1) \rightarrow W(n), n \geqslant 1$, for left-multiplication by $\mu_{0}$ (see Remark 1.23), and $e: W(n) \rightarrow W(n+1)$ for the inclusion.

Proposition 2.16. (i) The map $v_{1}$ is a chain-map of degree $q+1$. (ii) For $n>1$, it carries $W(n)$ into $W(n-1)$, and $v_{1} W(1) \subseteq W(1)$. (iii) For $n \geqslant 1, \bar{\mu}_{0} e=v_{1}: W(n) \rightarrow$ $W(n)$.

Proof. For (ii) note for $n=1$ that

$$
\lambda_{1} \Lambda(2 p-1)+\mu_{1} \Lambda(2 p+1) \subseteq \Lambda(3) \subseteq \Lambda(2 p+1)
$$

by admissibility; while, for $n>1$,

$$
\lambda_{1} \Lambda(2 p n-1)+\mu_{1} \Lambda(2 p n+1) \subseteq \Lambda(2 p(n-1)+1)
$$

by Proposition 1.18. For (iii), we note that, for $n \geqslant 1, \mu_{0} \lambda_{n+1} \equiv-\mu_{n} \lambda_{1}$ and $\mu_{0} \mu_{n+1} \equiv-\mu_{n} \mu_{1} \bmod \Lambda(2 n-1)$.

It would be interesting to have a geometric analogue of this result.

3. The Metastability Theorem. In this section, we prove our central result modulo several lemmas whose proofs are deferred to $\S 4$.

Theorem 3.1 (Metastability Theorem). For $1 \leqslant n \leqslant k \leqslant \infty$, the map induced in homology $H_{\sigma}^{s}$ by $W(n) \rightarrow W(k)$ is an isomorphism for

$$
\sigma<2\left(p^{2}-1\right)(s-2)+p q n-2 p-2
$$

and an epimorphism for

$$
\sigma \leqslant 2\left(p^{2}-1\right)(s-1)+p q n-2 p-2 .
$$

From the Localization Theorem stated in the Introduction (or from [6]), we see that $H_{\sigma}^{s}(M(0) \tilde{\otimes} \Lambda)=0$ for $\sigma<q s-1$, and so obtain from Theorem 3.1 the

Corollary 3.2 (Stability Theorem). For $1 \leqslant n \leqslant k \leqslant \infty$ the map induced in homology $H_{\sigma}^{s}$ by $\Lambda(2 n-1) \rightarrow \Lambda(2 k-1)$ is an isomorphism for

$$
\sigma<2(p-1)(s-2)+q n-3
$$

and an epimorphism for

$$
\sigma \leqslant 2(p-1)(s-1)+q n-3 .
$$


Since $v_{1}$-multiplication acts along a line eventually entering the "metastable range" described in Theorem 3.1, we have

Corollary 3.3. For $1 \leqslant n \leqslant k \leqslant \infty$ the map induced in homology by $W(n) \rightarrow$ $W(k)$ is an isomorphism after inverting $v_{1}$.

The proof of the Metastability Theorem closely parallels Mahowald's proof of the analogous result at $p=2$. To begin, consider the short exact sequence

$$
0 \rightarrow W(n) \stackrel{e}{\rightarrow} W(n+1) \stackrel{\hat{h}_{2}}{\rightarrow} F(n+1) \rightarrow 0
$$

in which we normalize the quotient so that $\left|\hat{h}_{2}\right|=(-1,-p q n+1)$. Thus, $F(n+1)$ has lower left corner at $(0,0)$; and the Metastability Theorem is equivalent to

THEOREM 3.5. $H_{\sigma}^{s} F(n+1)=0$ for $\sigma<2\left(p^{2}-1\right) s-2 p$.

Now, $F(n+1)$ can be written canonically but not differentially as

$$
F(n+1)=\bar{\lambda}_{n+1} \bigoplus_{j=0}^{p-1} \Sigma^{q j} W(p n+j) \oplus \bar{\mu}_{n+1} \bigoplus_{j=1}^{p} \Sigma^{q j} W(p n+j)
$$

where, as in (2.3) and (2.4), $\left|\bar{\lambda}_{n+1}\right|=(0,0)$ and $\left|\bar{\mu}_{n+1}\right|=(0,1)$. The injections $e^{j}$ : $W(p n) \rightarrow W(p n+j)$ then define a submodule

$$
\tilde{F}(n+1)=\bar{\lambda}_{n+1} \bigoplus_{j=0}^{p-1} \Sigma^{q j} W(p n) \oplus \bar{\mu}_{n+1} \bigoplus_{j=1}^{p} \Sigma^{q j} W(p n) .
$$

LEMMA 3.8. $\tilde{F}(n+1) \subset F(n+1)$ is a subcomplex.

We postpone the proof of this lemma. Note that an analogue of (2.8) gives rise to a short exact sequence

$$
0 \rightarrow \bar{\lambda}_{n+1} \frac{\Lambda(2 p(n+1)-1)}{\Lambda(2 p n-1)} \rightarrow F(n+1) \rightarrow \bar{\mu}_{n+1} \frac{\Lambda(2 p(n+1)+1)}{\Lambda(2 p n+1)} \rightarrow 0
$$

of chain complexes. We filter $F(n+1)$ by defining $F_{j}(n+1)$ as the image of $\bar{\lambda}_{n+1} \Lambda(2 p n+2 j-1) / \Lambda(2 p n-1)$ for $0 \leqslant j \leqslant p$, and as the pullback of $\bar{\mu}_{n+1} \lambda(2 p n+2(j-p)+1) / \Lambda(2 p n+1)$ for $p \leqslant j \leqslant 2 p$. Then

$$
F_{2 p}(n+1)=F(n+1) \text {, }
$$

and

$$
\begin{array}{cc}
E_{j}^{0} F(n+1)=\bar{\lambda}_{n+1} \Sigma^{q(j-1)} W(p n+j-1), & 0<j \leqslant p, \\
E_{j}^{0} F(n+1)=\bar{\mu}_{n+1} \Sigma^{q(j-p)} W(p n+j-p), & p<j \leqslant 2 p .
\end{array}
$$

Furthermore, the filtration restricts to a filtration of $\tilde{F}(n+1)$, and the inclusion $f$ : $\tilde{F}(n+1) \rightarrow F(n+1)$ induces the natural injections $e^{j}: W(p n) \rightarrow W(p n+j)$ on the $E^{0}$-level.

This "artificial" subcomplex $\tilde{F}(n+1)$ was introduced because its differential is "simple"; it can be analyzed completely by means of the inclusion relations (1.18). To describe the result of the analysis, let $A^{*}(1)$ denote the subalgebra of the Steenrod 
algebra generated by $\beta$ and $P^{1}$. It has a basis

$$
\left\{P^{i}, \beta P^{i}, P^{i+1} \beta, \beta P^{i+1} \beta: 0 \leqslant i<p\right\} \text {. }
$$

We may extend the multiplication $A^{*}(1) \otimes A^{*}(1) \rightarrow A^{*}(1)$ to an $A^{*}$-action $: A^{*} \otimes$ $A^{*}(1) \rightarrow A^{*}(1)$ by requiring

$$
P^{p} \cdot \beta=P^{p} \beta
$$

Let $M(1)$ be the dual right $A^{*}$-module, and let

$$
\left\{x_{q i}, x_{q i+1}, y_{q(i+1)+1}, y_{q(i+1)+2}: 0 \leqslant i<p\right\}
$$

be the basis dual to (3.12). Explicitly, the $A^{*}$-action is determined by

$$
\begin{gathered}
x_{q j+1} \beta=x_{q j}, \quad y_{q j+2} \beta=y_{q j+1}, \\
x_{q j} P^{1}=j x_{q(j-1)}, \quad x_{q j+1} P^{1}=(j-1) x_{q(j-1+1)}, \\
y_{q j+1} P^{1}=x_{q(j-1)+1}+j y_{q(j-1)+1}, \\
y_{q j+2} P^{1}=(j-1) y_{q(j-1)+2}, \quad y_{q p+1} P^{p}=x_{1} .
\end{gathered}
$$

Map $g: \tilde{F}(n+1) \rightarrow M(1) \tilde{\otimes} \Lambda$ by

$$
\begin{aligned}
\bar{\lambda}_{n+1} \bar{\lambda}_{p n+j} \nu \rightarrow x_{q j} \nu, & \bar{\lambda}_{n+1} \bar{\mu}_{p n+j} \nu \rightarrow x_{q j+1} \nu, \\
\bar{\mu}_{n+1} \bar{\lambda}_{p n+j} \nu \rightarrow-y_{q j+1} \nu, & \bar{\mu}_{n+1} \bar{\mu}_{p n+j} \nu \rightarrow y_{q j+2} \nu .
\end{aligned}
$$

LEMMA 3.17. The map $g: \tilde{F}(n+1) \rightarrow M(1) \tilde{\otimes} \Lambda$ is a chain-map, an injection, and an isomorphism in bidegrees $(s, \sigma)$ for which $\sigma<p^{2} q n-1$.

We defer the proof of this lemma also.

For $\sigma=p^{2} q n-1$, we have $x_{0} \otimes \lambda_{p^{2} n} \notin \operatorname{Im}(g)$ since the corresponding element $\bar{\lambda}_{n+1} \lambda_{p n} \lambda_{p^{2} n}$ is inadmissible.

The $A^{*}$-module $M(1)$ is naturally filtered by defining $M_{j}(1)$ by

$$
\begin{gathered}
M_{j}(1)=\left\langle x_{q i}, x_{q i+1}: i<j\right\rangle \text { for } 0 \leqslant j \leqslant p, \\
M_{j}(1)=M_{p}(1) \oplus\left\langle y_{q i+1}, y_{q i+2}: i \leqslant j-p\right\rangle \text { for } p<j \leqslant 2 p .
\end{gathered}
$$

If $M(1) \tilde{\otimes} \Lambda$ is filtered accordingly, then $g$ is filtration-preserving and, for each $j$, $E_{j}^{0}(g)$ is a suspension of the injection $e^{\infty}: W(q n) \rightarrow M(0) \tilde{\otimes} \Lambda$.

The final component of the proof is the following stable result, which follows from the proofs in [9].

THEOREM 3.20. $H_{\sigma}^{s}(M(1) \tilde{\otimes} \Lambda)=0$ for $\sigma<2\left(p^{2}-1\right) s-2 p$.

The obstruction to lowering the intercept here is the element $h_{11} h_{20}$.

The proof of Theorem 3.5 proceeds by induction on $\sigma$. The assertion is obvious for $\sigma<0$, so we pass to the inductive step: suppose (3.5) holds for all $n \geqslant 1, s \geqslant 0$, and $\sigma \geqslant 0$ with $\sigma<N$. Then:

(3.21) For any $j \geqslant 0, H_{\sigma}^{s} W(p n) \rightarrow H_{\sigma}^{s} W(p n+j)$ is an isomorphism for

$$
\sigma<2\left(p^{2}-1\right)(s-2)+p^{2} q n-2 p-2
$$

and an epimorphism for $\sigma \leqslant 2\left(p^{2}-1\right)(s-1)+p^{2} q n-2 p-2$ provided $\sigma<N$. 
We show that (3.5) is true for $\sigma<N+q-1$ by showing that the maps

$$
\begin{array}{llll} 
& H_{\sigma}^{s} \tilde{F}(n+1) & & \\
H_{\sigma}^{s} F(n+1) & \searrow g_{*} & \\
& & H_{\sigma}^{s}(M(1) \tilde{\otimes} \Lambda)
\end{array}
$$

are isomorphisms for $s, \sigma$ such that $\sigma<2\left(p^{2}-1\right) s-2 p$ and $\sigma<N+q-1$, and then appealing to Theorem 3.20 to see that $H_{\sigma}^{s}(M(1) \tilde{\otimes} \Lambda)$ is then trivial. The maps $f_{*}$ and $g_{*}$ are seen to be isomorphisms by induction on the filtrations. We deal with $f_{*}$ and leave $g_{*}$ to the reader.

To begin, $\tilde{F}_{1}(n+1) \rightarrow F_{1}(n+1)$ is an isomorphism. So let $1<j<2 p$ and suppose $H_{\sigma}^{s} \tilde{F}_{j-1}(n+1) \rightarrow H_{\sigma}^{s} F_{j-1}(n+1)$ is an isomorphism for $s, \sigma$ such that $\sigma<2\left(p^{2}-1\right) s-2 p$ and $\sigma<N+q-1$. We study the case $j \leqslant p$ and leave the rest to the reader. Form the map of long exact sequences associated to the diagram:

$$
\begin{aligned}
& 0 \rightarrow \tilde{F}_{j-1}(n+1) \quad \rightarrow \quad \tilde{F}_{j}(n+1) \quad \rightarrow \quad W(p n) \quad \rightarrow 0 \\
& \downarrow \quad \downarrow \quad \downarrow \\
& 0 \rightarrow F_{j-1}(n+1) \rightarrow F_{j}(n+1) \rightarrow W(p n+j-1) \rightarrow 0
\end{aligned}
$$

The inductive assumption and (3.21) feed into the 5-lemma to complete the induction in this case. The other cases are similar.

A computation through the 61 -stem at $p=3$ leads us to conclude that, in fact, a much stronger theorem is true. We propose the following:

CONJECTURE 3.22 (TERTIARY STABILITY). For $1 \leqslant n \leqslant k \leqslant \infty$ there are compatible maps

$$
\begin{array}{ccc}
H_{\sigma}^{s} F(n+1) & \rightarrow & H_{\sigma}^{s} F(k+1) \\
\searrow & & \swarrow \\
& H^{s}(M(1) \tilde{\otimes} \Lambda) &
\end{array}
$$

which are isomorphisms for

$$
\sigma<2\left(p^{3}-1\right)(s-2)+p^{2} q n-2\left(p^{2}+p+1\right)+1
$$

and epimorphisms for

$$
\sigma \leqslant 2\left(p^{3}-1\right)(s-1)+p^{2} q n-2\left(p^{2}+p+1\right)+1 .
$$

Does this pattern continue?

4. Deferred proofs. Lemmas 3.8 and 3.17 are proved together, by explicitly exhibiting the boundary $d$ of admissible generators in $\tilde{F}(n+1)$.

For instance, take

$$
\bar{\mu}_{n+1} \bar{\lambda}_{p n+j} \nu, \quad 1 \leqslant j \leqslant p, \nu \in \Lambda\left(2 p^{2} n-1\right) .
$$

In $W(n+1)$

$$
d\left(\bar{\mu}_{n+1} \lambda_{p n+j} \nu\right)=\bar{\mu}_{n+1} d\left(\lambda_{p n+j} \nu\right)-\bar{\lambda}_{n+1} \mu_{0} \lambda_{p n+j} \nu .
$$


In considering the first term here, we must compute $d\left(\lambda_{p n+j} \nu\right) \bmod \Lambda(2 p n-1)$. By (1.13) and (1.5),

$$
d \lambda_{p n+j}=\sum_{k} c_{p n+j, k} \lambda_{p n+j-k} \lambda_{k}
$$

where

$$
c_{p n+j, k} \neq 0 \Rightarrow p k<(p-1)(p n+j) .
$$

Claim 4.5. If (i) $k \geqslant j$ and (ii) $p k<(p-1)(p n+j)$ then

$$
\lambda_{p n+j-k} \lambda_{k} \Lambda\left(2 p^{2} n-1\right) \subseteq \Lambda(2 p n+1) .
$$

By Proposition 1.18, (i) guarantees that

$$
\lambda_{p n+j-k} \Lambda(2 p n+q(p n+j-k)) \subseteq \Lambda(2 p n),
$$

so it will be sufficient to prove

$$
\lambda_{k} \Lambda\left(2 p^{2} n-1\right) \subseteq \Lambda\left(2 p^{2} n-q(k-j)-1\right) .
$$

To see (4.7), we divide into two cases. If $2 p^{2} n-1<2 p k$, then, by admissibility

$$
\lambda_{k} \Lambda\left(2 p^{2} n-1\right) \subseteq \Lambda(2 k) .
$$

But (4.5)(ii) implies in this case that $q j \geqslant 2 p n$, so $2 p k<q(p n+j)=2 p^{2} n+q j-$ $2 p n \leqslant 2 p^{2} n-q(k-j)$. This establishes (4.7) in this case. If, on the other hand, $2 p^{2} n-1 \geqslant 2 p k$, then, by Proposition 1.18 again,

$$
\lambda_{k} \Lambda\left(2 p^{2} n-1\right) \subseteq \Lambda\left(2 p^{2} n-q k-1\right),
$$

and, as $j \geqslant 0,(4.7)$ is established in this case also. So Claim 4.5 is proved.

In considering the second term in (4.2), we must compute

$$
\mu_{0} \lambda_{p n+j} \nu \bmod \Lambda(2 p n-1) \text {. }
$$

By (1.9) and (1.5)

$$
\mu_{0} \lambda_{p n+j}=\sum_{k} c_{p n+j-1, k} \mu_{p n+j-k-1} \lambda_{k+1}
$$

where

$$
c_{p n+j-1, k} \neq 0 \Rightarrow p k<(p-1)(p n+j-1) .
$$

An argument similar to the above then shows

Claim 4.12. If (i) $k \geqslant j$ and (ii) $p k<(p-1)(p n+j-1)$, then

$$
\mu_{p n+j-k-1} \lambda_{k+1} \Lambda\left(2 p^{2} n-1\right) \subseteq \Lambda(2 p n-1) .
$$

Combining the two claims, we find that in $F(n+1)$

$$
\begin{aligned}
d\left(\bar{\mu}_{n+1} \bar{\lambda}_{p n+j} \nu\right)= & \sum_{k=1}^{j-1} c_{p n+j, k} \bar{\mu}_{n+1} \bar{\lambda}_{p n+j-k} \lambda_{k} \nu-\bar{\mu}_{n+1} \bar{\lambda}_{p n+j} d \nu \\
& -\sum_{k=0}^{j-1} c_{p n+j-1, k} \bar{\lambda}_{n+1} \bar{\mu}_{p n+j-k-1} \lambda_{k+1} \nu .
\end{aligned}
$$


Note that, by (4.7), each term in the first sum lies in $\tilde{F}(n+1)$. Similarly, one sees that each term in the last term lies in $\tilde{F}(n+1)$; and since $d \nu \in \Lambda\left(2 p^{2} n-1\right)$, the remaining term does also. This proves Lemma 3.8 in this instance, and the others are similar. This completes the proof of Lemma 3.8.

For Lemma 3.17, an easy computation with binomial coefficients shows that

$$
\begin{array}{cc}
c_{p n+j, k}=-\left(\begin{array}{l}
j \\
k
\end{array}\right) & \text { for } k \leqslant j<p, \\
d_{p n+j, k}=\left(\begin{array}{c}
j-1 \\
k
\end{array}\right) & \text { for } k<j<p .
\end{array}
$$

This simplifies (4.13) and its analogues.

A series of similar arguments may now be summarized as follows:

Proposition 4.16. In $\tilde{F}(n+1)$,

$$
\begin{aligned}
d\left(\bar{\lambda}_{n+1} \bar{\lambda}_{p n+j} \nu\right) & =\sum_{k=1}^{j}\left(\begin{array}{l}
j \\
k
\end{array}\right) \bar{\lambda}_{n+1} \bar{\lambda}_{p n+j-k} \lambda_{k} \nu+\bar{\lambda}_{n+1} \bar{\lambda}_{p n+j} d \nu \\
d\left(\bar{\lambda}_{n+1} \bar{\mu}_{p n+j} \nu\right)= & \sum_{k=0}^{j}\left(\begin{array}{c}
j \\
k
\end{array}\right) \bar{\lambda}_{n+1} \bar{\lambda}_{p n+j-k} \mu_{k} \nu \\
& -\sum_{k=1}^{j-1}\left(\begin{array}{c}
j-1 \\
k
\end{array}\right) \bar{\lambda}_{n+1} \bar{\mu}_{p n+j-k} \lambda_{k} \nu-\bar{\lambda}_{n+1} \bar{\mu}_{p n+j} d \nu \\
d\left(\bar{\mu}_{n+1} \bar{\lambda}_{p n+j} \nu\right)= & \sum_{k=0}^{j-1}\left(\begin{array}{c}
j-1 \\
k
\end{array}\right) \bar{\lambda}_{n+1} \bar{\mu}_{p n+j-k-1} \lambda_{k+1} \nu \\
& -\sum_{k=0}^{j}\left(\begin{array}{c}
j \\
k
\end{array}\right) \bar{\mu}_{n+1} \bar{\lambda}_{p n+j-k} \lambda_{k} \nu-\bar{\mu}_{n+1} \bar{\lambda}_{p n+j} d \nu \\
d\left(\bar{\mu}_{n+1} \bar{\mu}_{p n+j} \nu\right)= & \sum_{k=0}^{j-1}\left(\begin{array}{c}
j-1 \\
k
\end{array}\right) \bar{\lambda}_{n+1} \bar{\mu}_{p n+j-k-1} \mu_{k+1} \nu \\
& -\sum_{k=0}^{j}\left(\begin{array}{c}
j \\
k
\end{array}\right) \bar{\mu}_{n+1} \bar{\lambda}_{p n+j-k} \mu_{k} \nu \\
& +\sum_{k=1}^{j-1}\left(\begin{array}{c}
j-1 \\
k
\end{array}\right) \bar{\mu}_{n+1} \bar{\mu}_{p n+j-k} \lambda_{k} \nu+\bar{\mu}_{n+1} \bar{\mu}_{p n+j} d \nu .
\end{aligned}
$$

Lemma 3.17 now follows from this by combining (3.15) with (2.13).

\section{REFERENCES}

1. J. F. Adams, On the groups $J(X)$. IV, Topology 5 (1966), 21-71.

2. A. K. Bousfield et al., The mod-p lower central series and the Adams spectral sequence, Topology 5 (1966), 331-342.

3. A. K. Bousfield and D. M. Kan, The homotopy spectral sequence of a space with coefficients in a ring, Topology 11 (1977), 79- 106.

4. J. Harper, Rank $2 \bmod 3 \mathrm{H}$-spaces, Canad. Math. Soc. Conf. Proc., Current Trends in Algebraic Topology, Western Ontario, 1981. 
5. D. C. Johnson, H. R. Miller, W. S. Wilson and R. S. Zahler, Boundary homomorphisms in the generalized Adams spectral sequence and the nontriviality of infinitely many $\gamma_{t}$ in stable homotopy, Notas de Mat. y Symp., No. 1: Reunion Sobre Teoria de Homotopia, Northwestern Univ., Soc. Mat. Mex., 1975, pp. 47-59.

6. A. Liulevicius, Zeroes of the cohomology of the Steenrod algebra, Proc. Amer. Math. Soc. 14 (1963), 972-976.

7. M. Mahowald, On the double suspension homomorphism, Trans. Amer. Math. Soc. 214 (1975), $169-178$.

8. H. R. Miller, A localization theorem in homological algebra, Math. Proc. Cambridge Philos. Soc. 84 (1978), 73-84.

9. H. R. Miller and C. Wilkerson, Vanishing lines for modules over the Steenrod algebra, J. Pure Appl. Algebra 22 (1981), 293-308.

10. M. C. Tangora, Some remarks on the lambda algebra, Geometric Applications of Homotopy Theory. II, (Proceedings, Evanston 1977), Lecture Notes in Math., vol. 658, Springer-Verlag, Berlin and New York, 1978, pp. 476-487.

11. F. R. Cohen, The unstable decomposition of $\Omega^{2} \Sigma^{2} X$ and its applications (to appear).

DePARTMENT OF MATHEMATICS, UNIVERSITy OF ROCHESTER, ROCHESTER, NeW YORK 14620

Department of Mathematics, University of Washington, Seattle, Washington 98195 